\title{
Design and Implementation of Computer Hardware Monitoring System Based on Cloud Computing
}

\author{
Ming Liu, Ping Guo \\ Kunming University of Science and Technology
}

\begin{abstract}
From the perspective of customer service and on the basis of network, this paper proposes a computer hardware monitoring system based on cloud platform, which separates the data acquisition, storage, and analysis based on cloud computing hardware monitoring system. The computer hardware monitoring system based on cloud platform separates data storage and access. The cloud platform unifies modeling analysis of storage hardware parameters for huge amounts of data, in order to provide users with complete hardware maintenance information. This paper introduces the overall design of the hardware monitoring system, and then gives the system structure and related key technologies of implementation instance.
\end{abstract}

Key word: computer hardware monitoring system; cloud computing;

\section{Introduction}

Monitoring computer hardware running parameters is very important to hardware maintenance. During hardware temperature monitoring, the high temperature leads to unstable running of the computer system, and even affecting the service life of the equipment. Therefore, hardware parameters monitoring is particularly important in unattended server rooms, cluster servers and massive cloud server platforms. Desktop computers, notebook computers, smart phones, tablets or a intelligent terminal that can run program are often produced by different manufacturers having very different hardware composition and production process. It is difficult to analyze effectively the health status of the hardware device. We can assess more effectively the health status of the hardware and do timely maintenance if we can do horizontal and vertical modeling analysis of the historical data of the various parameters of hardware, and the health status data of the similar hardware equipment.

In addition, most of the existing enterprises or schools establish computer room environment monitoring systems to build computer hardware parameters such as temperature monitoring. The defect is that a considerable investment of hardware is required to run and cannot directly detect the temperature of the computer and various states and parameters. At the same time it can't make full use of the resources of existing computer system itself. All sorts of computing device and intelligent terminal maintenance often is unable to browse the user's history of hardware running parameters. This also brings great inconvenience for the hardware maintenance.

In this paper, from the user service point of view and on the basis of network monitoring parameters, we propose a computer hardware monitoring system based on cloud platform. It is a cloud computing hardware monitoring system that separates the data acquisition and storage management. The computer hardware monitoring system based on cloud platform separates data storage and access. Based on the platform it unifies modeling analysis of storage hardware parameters of huge amounts of data, so as to provide users with good hardware maintenance information. This paper introduces the overall design of the hardware monitoring system, and then gives the system structure and related key technologies of implementation instance.

\section{System composition}

Through the monitoring software to obtain the effective parameters by monitoring operating parameters of a variety of computer hardware, operating parameters and related software and through the network timing to the monitoring server to send all available; monitoring server in the cloud gets and statistics, analysis and processing all kinds of parameter information, unified algorithm, based on information of various parameters determined by monitoring computer hardware health state and save the parameter and state, promptly notify the users of health status. The client component can be composed of three parts: the client component, the cloud service module and the communication network. The client component is run on a monitored computer or intelligent terminal, and the server component is deployed on the monitor server cluster.

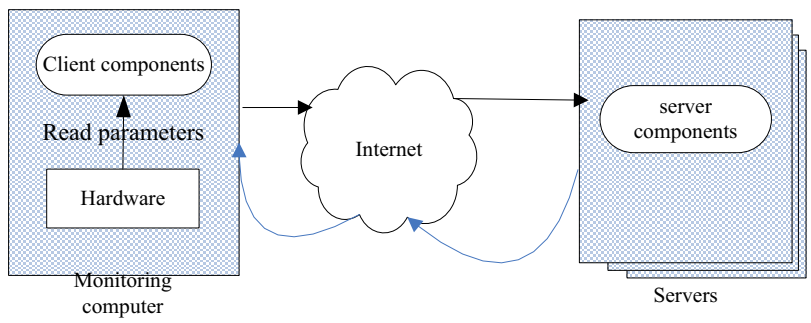

Figure 1

Corresponding author: kgliuming@qq.com 
As shown in Figure 1, the entire system consists of three parts: client components, server components and communication networks.

\section{Communication protocol design}

The basic work process is that the client components read all kinds of hardware and operating parameters of the machine, and the parameters are transmitted to the monitoring server, and the running parameters are processed by the monitoring server in the cloud. The UDP/IP protocol is used as the basic communication protocol between the client and the server. When it is transmitted in the network, the communication protocol layer is as follows:

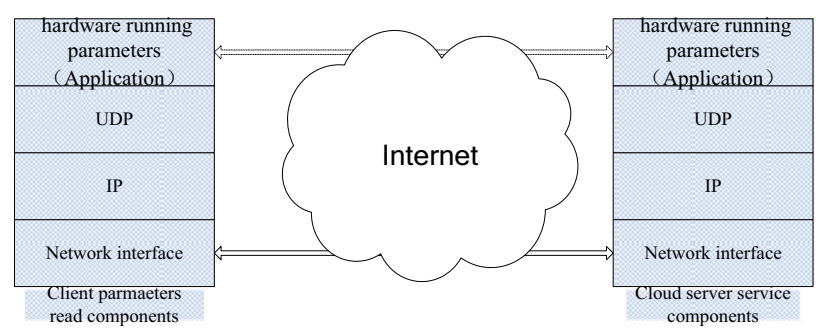

Figure 2

Monitoring information data packet format is as follows: the underlying data unit is 8 bytes, a data unit that represents a parameter value of a hardware device. The hardware device number is 1 bytes, the value range of $0 \sim 255$, it can express 256 kinds of hardware, such as the agreement in the provisions of 1, express CPU, 2 express the memory, 3, said the hard disk, etc. The parameter type is a byte, range is from 0 to 255 , can be expressed 256 types of parameters, such as agreement provisions 1 represent temperature 2 said fan speed, three voltage and 4 represents the frequency and 5 representing $\mathrm{CPU}$ utilization. The parameter value is 6 bytes, the first 4 bytes represent the integer part of the parameter, and the next two bytes represent the decimal part of the parameter. The communication protocol to UDP/IP protocol data transmission

\section{Process design}

The process design of computer client components

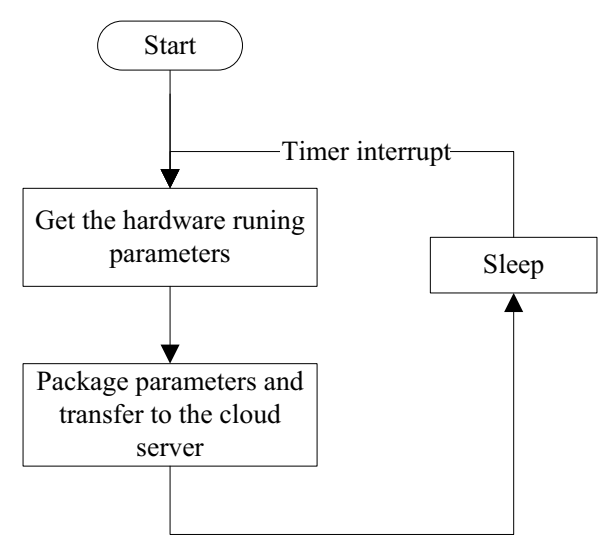

Figure 3

Set the relevant operating parameters, including the server IP address, port number and the time interval to send data to the server. Port number must be consistent with the server, usually set the server port number, and then according to the port number of the server, to set the port number of the reading software. Start the software, the software is set to start with the operating system to start. Read the storage server IP address and timer time interval. IP address can be set in the program and do not have to set. The default timer interval is $60 \mathrm{~s}$, can be changed according to the actual situation.

Client components to wait for the timer time to. When the timer time, read the relevant hardware sensor parameters and other operating parameters. Send the read data to the server through the UDP/IP protocol.

The process design of cloud server's service component

The cloud service component process is first set up a listener port number on the server side, and the step is only carried out when the first operation is carried out. After setting, this step can be ignored. Service software can set a default port number, if you do not manually set, then the software automatically takes the default port number. After starting the cloud service component, the service component gets the port number of the set listening port. The cloud server's service component listening the port. When there are new data packets, analysis of the data package, mainly including: validity verification, access to each other's IP address, access to the other side of the host name and hardware operating parameters. According to the host name and IP address to determine whether the client has been listening in the host library, if there is no increase in the host information, if there is to update the host state and saving

\section{Parameter reading}

Currently computer system motherboards, CPU and hard drive and other hardware devices are equipped with sensors, can provides CPU temperature, CPU voltage, CPU fan speed, the temperature inside the host and hard disk also provides the hard disk parameters such as temperature and hardware vendors provide the read sensor data access interface parameters can be provided 
by the operating system I / O interface function read out the motherboard BIOS stored in a variety of sensors, realtime data, important parameters are: temperature of CPU, CPU voltage, CPU fan speed, the temperature inside the host, hard disk temperature. According to the need, you can also read the operating system to provide the CPU usage and other parameters.

Currently client system motherboard, CPU and hard drive and other hardware devices are integrated sensors, can provides CPU temperature, CPU voltage, CPU fan speed, mainframe temperature, as well as the hard disk also provides the hard disk parameters such as temperature and hardware vendors provide read related sensor data access interface. The basic method is to read the real time parameters of various sensors stored in the hardware through the API functions provided by the operating system. Such as in the windows system can first call API function CreateFile to open the device and returns a handle to a device associated with. Then, and then call the API function DeviceIoControl function and driving communication program, and then read the various parameters of the equipment. The basic method to obtain the operating parameters of the operating system is to call the interface functions provided by the operating system to complete the.

In the client components, in addition to reading the hardware sensor parameters, but also can easily read the operating system to provide a variety of parameters, such as the use of CPU and other important parameters. According to the actual demand, you can also read some important services, such as Windows system software, including Server SQL and other running state parameters for these servers to monitor.

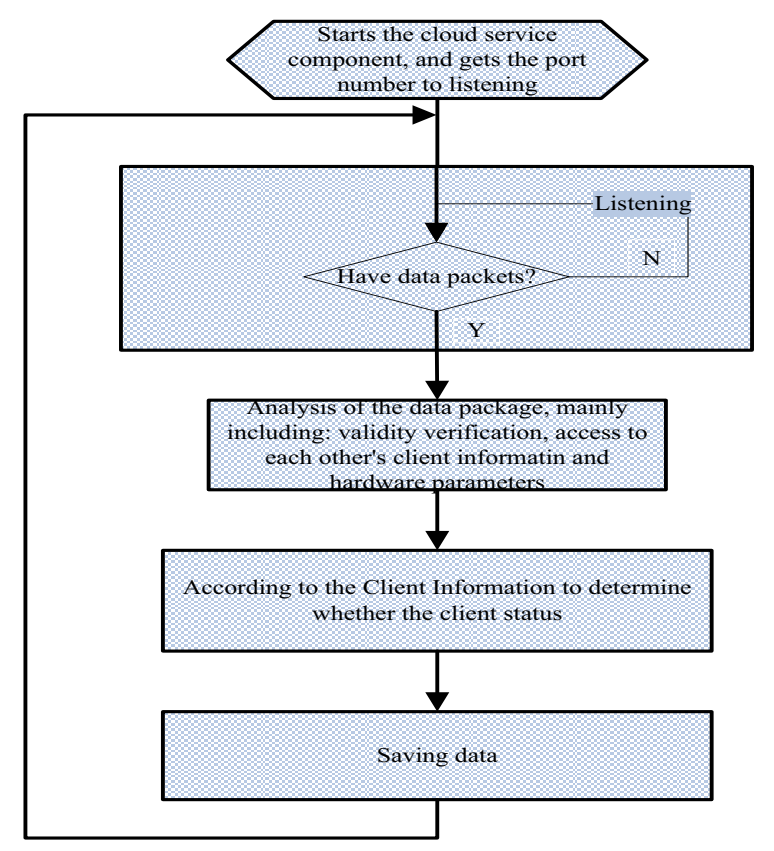

The core function of the server component is on the network resource management and scheduling, i.e. resource scheduling, allocation and recovery work. This is the resource management and scheduling module to realize the function of this layer, the kernel function is similar to the traditional stand-alone operating system. The requirements for scheduling and management of network resources to meet the needs of different the need for different attributes of different resources, strategies and methods of scheduling, allocation and recovery algorithm based on the realization of resources on demand elasticity flow. The server component also has interface with the interface module is used to realize the interaction with the user, receive and complete from the upper application and user requests. The server has two special the module, network communication and optimization module and network communication and service protocol module. The client network communication and optimization module mainly completes Interrupt start terminal interception, and the signal is transmitted to the web server, by the server to deal with. Network communication and optimization module of the server end will respond to requests from the client. The first is a network address assigned to the client, to facilitate the subsequent communication; secondly, it will on the user's identity authentication and will return to support network autonomous system example list of operating systems to carry on a choice to the user; after users choose what you want to run an operating system instance, and then call the relevant transmission protocol will part of the data to the terminal, client interaction.

Virtual resource server component driver module, is the network based on interrupt management, complete the driver and the management of virtual resources. Including the following three functions: (1) on the physical hardware, such as graphics card, such as driving, driving after all hardware devices autonomous network system in (2) in the real hardware drive based on the further formation of virtual resources, such as the virtual CPU, virtual memory, virtual memory, virtual equipment resources construction, driving and management; (3) implementation of large-scale data storage, network storage and retrieval of large data. Resource storage and management module is based on virtual network storage, further examples of the operating system, applications and data organization, storage and management for users efficiently Visit. Based on cloud computing hardware monitoring system hardware resources are divided into three categories: the first is composed of terminal, server and network hardware platform resources. Therefore, with the traditional operating system only management and scheduling applications

\section{The analysis of communication efficiency}

Monitoring system is often run on the existing network, in order not to affect the current system of CPU usage, and the characteristics of the variation of parameters such 
as temperature, the timing of the client components can be as long as possible, the default is $10 \mathrm{~s}$. General recommendations are set to $30 \mathrm{~s}$.

At the same time, as far as possible to reduce network traffic. For the monitoring system, the amount of information is small. In order to reduce the impact of the current network traffic, the invention adopts the UDP/IP protocol between the host and the server. IP protocol related standards documents, the host or network device IP data reported length shall not be less than 576 bytes, the first 20 bytes of IP, the maximum length of 40 bytes. That is to say, IP net charge at least 516 bytes length, and then deduct the UDP protocol header 8 bytes, 508 bytes. It can be estimated that the basic data unit of each transmission monitoring information can be completed in a data packet in the network when no more than 56 units are available. Generally can be read in the BIOS hardware operating parameters have 30 or so, which is an important CPU temperature, CPU fan speed, chassis system temperature and other key parameters. Therefore, it can ensure the monitoring information in a packet to complete the transmission, there is no case of split data packets. Data does not exist in the case of sub packets, you can consider the use of the UDP protocol such a shortcut to reduce the impact of the current network traffic as much as possible.

\section{Conclusion}

The hardware monitoring system based on cloud computing is proposed in this paper. Through regularly reading all kinds of parameters of the sensor computing devices, and then transmitted to the cloud to store and analyze data, it can determine the health status of computer equipments. It has a good expansibility. It can monitor more detailed computer operation parameters. It can better and more effectively define computer running states. It is convenient for monitoring, management and analysis of hardware faults,. It can more effectively maintain equipment. In this paper, the hardware parameters monitoring system based on cloud computing can effectively reduce the cost of equipment maintenance.

\section{References}

1. Michael Armbrust, Armando Fox, Rean Griffith, Anthony D.Joseph, Randy Katz, Andy Konwinski, Gunho Lee, David Patterson, Ariel Rabkin, Ion Stoica, Matei Zaharia. A view of cloud computing [ $\mathrm{J}$ ] . Communications of the ACM, 2010, 53( 4) : 50- 58.

2. Fay Chang, Jeffrey Dean, Sanjay Ghemawat, et al. Bigtable: A distributed storage system for structured data[ A] . Proc of the Seventh Sympo sium on Operating System Design and Implementation ( OSDIT06) [ C] . Seattle,WA : USENIX, 2006. 205-218.

3. David Chappell. Introducing the Azure Services Platform[ EB/OL] . http: //download. microsoft. com/ . . . / e/ . . . / Azure-Services- Platform. docx, 2011-03-18.
4. [4] Dhruba Borthakur. The Hadoop Distributed File System: Architecture and Design[ EB/ OL] . http: / / hadoop. apache. org/ 